[Agr. Biol. Chem., Vol. 36, No. 4. p. 639 644, 1972]

\title{
Studies on the Chemical Structure of Konjac Mamnan
}

\author{
Part III. Theoretical Aspect of Controlled Degradation of \\ the Main Chain of the Mannan ${ }^{\dagger}$
}

\author{
By Koji Kato* and Kazuo Matsuda \\ Department of Agricultural Chemistry, Faculty of Agriculture, \\ Tohoku University, Sendai
}

Received October 1, 1971

\begin{abstract}
Rate-constants of $\beta$-D-mannosidic and $\beta$-D-glucosidic linkages for acid hydrolysis were determined, And from these results, statistical analysis of acid hydrolysis of the main chain of the mannan was made whereby the yields of the fragmental disaccharides could be calculated for any degree of hydrolysis. The symmetry of the result of these calculations with those observed experimentally may support the proposed chemical structure of the mannan. Moreover, from the result of action of cellulase on the manno-oligosaccharides, the main process of the reaction, "hydrolysis of the mannan by cellulase" was discussed.
\end{abstract}

Previous publications ${ }^{1,21}$ described some oligosaccharides obtained from acid and enzymatic hydrolyzates of the mannan. And on the strength of these results, the authors presumed the repeating unit of the main chain of the mannan. These oligosaccharides had been considerably different in yields, which would be assumed to be in proportion to both the number of the oligosaccharide-unit and the resistivity of the glycosidic linkages in the polymer. Therefore, if the glucomannan has the chemical structure mentioned in Part $\mathrm{II}^{2}{ }^{2)}$ information on the dependences of the nature and the yields of the fragmental disaccharides upon the degree of depolymerization can be obtained from a statistical treatment of the reaction.

When we obtain a disaccharide by partial

1 Preliminary reports of the experimental portion of this work appeared in ref. 1) and 2).

* Present address: Department of Agricultural Chemistry, Faculty of Agriculture, Shizuoka University, Iwata, Shizuoka Prefecture.

The abbreviations used are: $M, D-m a n n o p y r a n o s y l$ residue; G, D-glucopyranosyl residue; $\rightarrow 3-1,4$ glycosidic linkage. acid hydrolysis of a polysaccharide, some molecules of the disaccharide are produced directly from the polysaccharide but the others are from the higher oligosaccharides containing the disaccharide residue. Since the disaccharide thus formed is immediately converted by acid to constitutional monosaccharide, an amount of the disaccharide in the reaction mixture is not calculated precisely, unless rate-constants of each process are determined. Accordingly, we attempted to simplify the calculation with the following assumptions;

1) All of the same glycosidic linkages in a polysaccharide are hydrolyzed regardless of their position in the chain: The effect of terminal units is neglected on the basis of assumption that the original chains are sufficiently long.

2) A ratio of resistivities of $\beta-1,4$ mannobiose and $\beta-1,4$ glucobiose (cellobiose) towards acid is regarded as a ratio of those of $\beta$-D-mannosidic acid $\beta$-D-glucosidic linkages in the polysaccharide.

Acid catalyzed hydrolysis of glycosidic linkages is known to be a first-order reaction. Supposing that initial (time 0 ) concentration of 
the polysaccharide (including oligosaccharides except disaccharides) is $a$, and that $x$ fraction of the polymer is hydrolyzed during time $t$, remaining concentration of the polymer is shown as $(a-x)$. In the above case, decreasing rate of the polymer is in proportion to a concentration of the polymer at that time. This is consequently formulated as follows;

$$
(a-x)=a \cdot e^{-k t}
$$

Since we can use the number of the glycosidic linkage in the polysaccharide instead of a concentration of the polysaccharide, let $G_{t}$ and $M_{t}$ be the numbers of $\beta$-D-glucosidic and $\beta$ $D$-mannosidic linkages remaining at time $t$, respectively. And let the rate-constants for the hydrolysis of $\beta$-D-glucosidic and $\beta$-D-mannosidic linkages be $k$ and $2.5 k$ (see Experimental), respectively. Then

$$
\begin{aligned}
G_{t} & =G_{0} \cdot e^{-k t} \\
M_{l} & =M_{0} \cdot e^{-2.5 k t}
\end{aligned}
$$

where $G_{0}$ and $M_{0}$ stand for the number of $\beta$-D-glucosidic and $\beta$-D-mannosidic linkages at $t=0$, respectively. The fraction of remaining linkages at time $t$ is shown as follows;

$$
\begin{gathered}
G_{t} / G_{0}=e^{-k t}=P_{g} \\
M_{t} / M_{0}=e^{-2.5 k t}=P_{m}
\end{gathered}
$$

Therefore we obtain

$$
P_{g}^{2.5}=P_{m}
$$

Since these fractions, $P_{g}$ and $P_{m}$, also indicate the probabilities of survival of $\beta$-Dglucosidic and $\beta$-D-mannosidic linkages during hydrolysis, the probabilities of the cleavage of these linkages would be $\left(1-P_{g}\right)$ and $\left(1-P_{m}\right)$, respectively.

If all of the D-glucose residue in the glucomannan take part in the constitution of cellobiose $(G \rightarrow G)$ unit, then a part of the structure of the glucomannan is formulated as follows;

$$
\rightarrow \mathrm{M} \rightarrow \mathrm{G} \rightarrow \mathrm{G} \rightarrow \mathrm{M} \rightarrow
$$$$
\text { A B C }
$$

In order to produce $\mathrm{G} \rightarrow \mathrm{G}$ from the above glucomannan, linkage $A$ and $C$ must be hydrolyzed and their probabilities are $\left(1-P_{m}\right)$ and $\left(1-P_{g}\right)$, respectively. Furthermore, linkage $B$ must survive the cleavage, and its probability is $P_{g}$. Therefore, the probability of obtaining $\mathrm{G} \rightarrow \mathrm{G}$ is

$$
\left(1-P_{m}\right) \cdot P_{g} \cdot\left(1-P_{g}\right)=P_{g}\left(1-P_{g}\right)\left(1-P_{g}{ }^{2.5}\right)
$$

Likely, the probability of obtaining $\mathrm{G} \rightarrow \mathrm{M}$ is calculated as

$$
\left(1-P_{g}\right) \cdot P_{g} \cdot\left(1-P_{m}\right)=P_{g}\left(1-P_{g}\right)\left(1-P_{g}^{2.5}\right)
$$

Simplified calculation thus shows that if all of the D-glucose residue constitute the cellobiose unit, ${ }^{11}$ the yields of cellobiose and epicellobiose $(\mathrm{G} \rightarrow \mathrm{M})$ should be almost equal unless any steric or energetic factors operate in such a way as to favor the preferential formation of the either one. However, when D-glucose residue is interposed as single unit between D-mannose residues, the structure of the glucomannan is partially shown as $\rightarrow M \rightarrow G \rightarrow M \rightarrow$. Therefore, probability of obtaining $\mathrm{G} \rightarrow \mathrm{M}$ from this part is $\left(1-P_{m}\right) P_{g}\left(1-P_{m}\right)$, namely $P_{g}\left(1-P_{g}{ }^{2.5}\right)^{2}$ Consequently, the total probability of obtaining $\mathrm{G} \rightarrow \mathrm{M}$ becomes

$$
P_{g}\left(1-P_{g}\right)\left(1-P_{g}^{2.5}\right)+P_{g}\left(1-P_{g}^{2.5}\right)^{2}
$$

Furthermore, $\left(G_{0}+M_{0}\right)$ is the sum of $\beta$-Dglucosidic and $\beta$-D-mannosidic linkages in the polysaccharide. The numbers of $\beta$-D-glucosidic and $\beta$-D-mannosidic linkages at time $t$ are $G_{0} \cdot P_{g}\left(=G_{t}\right)$ and $M_{0} \cdot P_{m}\left(=M_{t}\right)$, respectively. Therefore, the ratio of glycosidic linkages servived acid hydrolysis to the originally existed glycosidic linkages in the polysaccharide is shown as follows;

$$
\frac{P_{g} G_{0}+P_{m} M_{0}}{G_{0}+M_{0}}=\frac{P_{g}\left(G_{0} / M_{0}\right)+P_{m}}{G_{0} / M_{0}+1}
$$

If we adopt the ratio $3 / 5$ as $G / M$ in place of $G_{0} / M_{0}$, the degree of hydrolysis is shown as follows;

$$
\left(1-\frac{3 P_{g}+5 P_{m}}{8}\right) \times 100(\%)
$$




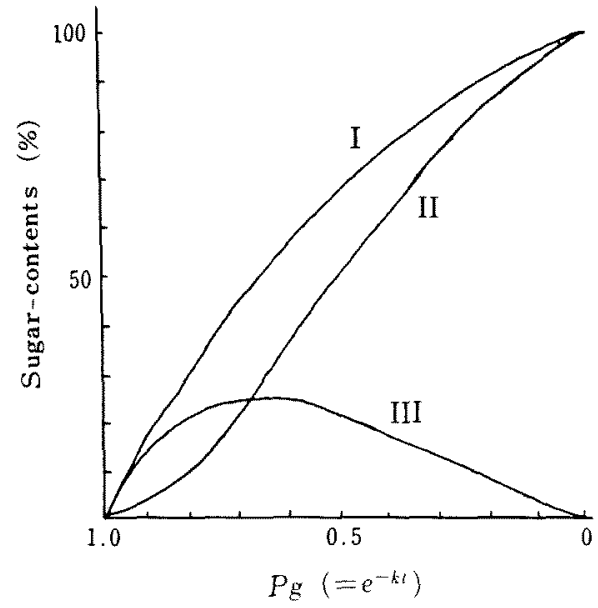

FIG. 1. Theoretical Curves of Partial Acid Hydrolysis.

I. Degree of hydrolysis (eq. (1) in the text)

II. Monosaccharides-content (1/8 eq. (2) in the text)

III. Oligosaccharides-content (III $=$ I - II $)$

The following repeating unit was postulated as the main chain of the glucomannan; ${ }^{21}$

$$
\begin{aligned}
& \mathrm{A}: \rightarrow \mathrm{G} \rightarrow \mathrm{G} \rightarrow \mathrm{M} \rightarrow \mathrm{M} \rightarrow \mathrm{M} \rightarrow \mathrm{M} \rightarrow \mathrm{G} \rightarrow \mathrm{M} \rightarrow \\
& \mathrm{B}: \quad \rightarrow \mathrm{M} \rightarrow \mathrm{G} \rightarrow \mathrm{M} \rightarrow \mathrm{M} \rightarrow \mathrm{M} \rightarrow \mathrm{M} \rightarrow \mathrm{G} \rightarrow \mathrm{G} \rightarrow
\end{aligned}
$$

In both cases, the probability of the presence of monosaccharides in the reaction mixture is shown, in the same manner as in the case of $\mathrm{G} \rightarrow \mathrm{M}$, as follows;

$$
3 P_{g}^{5}+4 P_{g}^{3.5}-10 P_{g}^{2.5}+P_{g}{ }^{2}-6 P_{g}+8
$$

Since $P_{9}$ varies from 1 (time 0 ) to 0 (hydrolyzed completely) with reaction time, the ratio of the monosaccharides in the hydrolyzate at each reaction time to those of the mixture hydrolyzed completely can be obtained as a quotient of eq. (2) divided by eight which is a numeral obtained at $P_{g}=0$. Curves provided by $1 / 8 \cdot$ eq. (2) and eq. (1) are depicted in Fig. 1. $\{$ Eq. (1) $-1 / 8$ eq. (2)\} is the probability of obtaining the whole oligosaccharides at each time, and its curve is shown as III in Fig. 1. The amount of oligosaccharides in the hydrolyzate will reach the maximum between $35 \%$
$\left(P_{g}=0.8\right)$ and $65 \%\left(P_{g}=0.55\right)$ of the degree of hydrolysis. The degree of hydrolysis (35\%) in fragmentation analysis by acid hydrolysis ${ }^{11}$ does not conflict with the above idea.

Furthermore, the equations of the probabilities of obtaining each disaccharide from the repeating unit are obtained in the same manner;

$$
\begin{array}{ll}
\text { G-M: } & P_{g}\left(1-P_{g}{ }^{2.5}\right)\left(2-P_{g}-P_{g}{ }^{2.5}\right) \\
\text { G-G: } & P_{g}\left(1-P_{g}\right)\left(1-P_{g}{ }^{2.5}\right) \\
\text { M-M: } & P_{g}{ }^{2.5}\left(1-P_{g}{ }^{2.5}\right)\left(3-P_{g}-2 P_{g}^{2.5}\right) \\
\text { M-G: } & P_{g}{ }^{2.5}\left(1-P_{g}\right)\left(2-P_{g}-P_{g}{ }^{2.5}\right)
\end{array}
$$

Thirty-five percent of hydrolysis of the glucomannan corresponds to about 0.8 of $P_{g}$. Therefore, the probabilities of each disaccharide are 0.214 for $G \rightarrow M, 0.068$ for $G \rightarrow G, 0.256$ for $M \rightarrow M$ and 0.070 for $M \rightarrow G$. This indicates that the yields of each disaccharide will be $21.4,6.8,25.6$ and $7 \%$ of the theoretical pos sible maximum, respectively. In the case of $\mathrm{G} \rightarrow \mathrm{M}$, calculation of theoretical yield is as follows; Two moles of $\mathrm{G} \rightarrow \mathrm{M}$ are produced from one mole of repeating unit (glucomannan). Since the molecular weights of $\mathrm{G} \rightarrow \mathrm{M}$ and the repeating unit are 342 and $1296(=162 \times 8)$, respectively, the theoretical yield of $G \rightarrow M$ based on the repeating unit is shown as $342 \times 2 / 1296=0.528$. Therefore, the amount of $\mathrm{G} \rightarrow \mathrm{M}$ at $35 \%$ of hydrolysis would be $0.528 \times$ $0.214=0.113$ of the original glucomannan. The same calculations are made for each disaccharide in consideration that in the case of $M \rightarrow M$, three moles of the sugar are not formed concurrently from one mole of repeating unit. Curves based on such calculations are depicted in Fig. 2.

The calculated and the actual yields of each disaccharide from $80 \mathrm{~g}$ of the glucomannan are shown in Table I. The ratio of $G \rightarrow G$ to $\mathrm{G} \rightarrow \mathrm{M}$ is in good agreement with experimental result, although those of $M \rightarrow M$ and $M \rightarrow G$ to $\mathrm{G} \rightarrow \mathrm{M}$ are for apart from experimental result. This may indicate that the assumption (2) is not completely correct, or else that $\beta$-D-mannosidic and $\beta$-D-glucosidic linkages in the 
polymer are hydrolyzed dependently.

Difference of the calculated yields from the

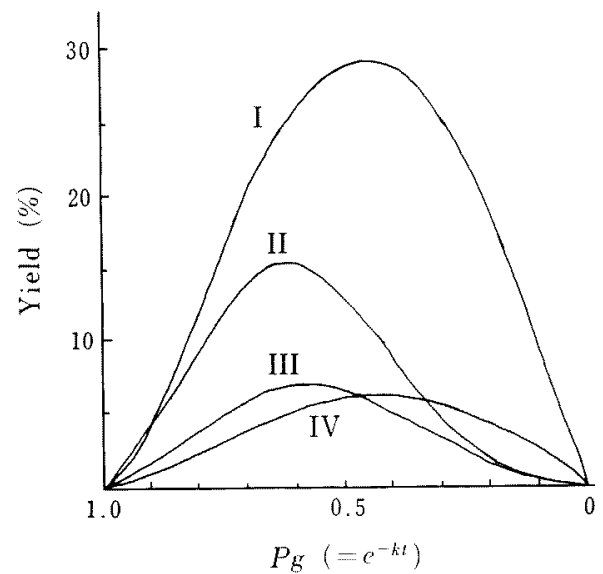

FIG. 2. Theoretical Yields of the Disaccharides from the Mannan.

I. $\mathrm{G} \rightarrow \mathrm{M}$ (Epicellobiose)

II. $\mathrm{M} \rightarrow \mathrm{M}$ (Mannobiose)

III. $M \rightarrow G$ (Mannosylglucose)

IV. $G \rightarrow G$ (cellobiose) actual yields will be explained by the fact that rate-constants of the disaccharides and the polysaccharide for acid hydrolysis are much more different. As the matter of fact, it has been reported that cellobiose is hydrolyzed about three times as fast as cellulose itself. $^{3 \prime}$

Secondly, in order to presume the position of the glucomannan attacked in the main by cellulase, the manno-oligosaccharides, $M \rightarrow M$, $\mathrm{M} \rightarrow \mathrm{M} \rightarrow \mathrm{M}$ and $\mathrm{M} \rightarrow \mathrm{M} \rightarrow \mathrm{M} \rightarrow \mathrm{M}$, were treated

Table. I. Calculated and Experimental Yields of THE Disaccharides Obtained From $80 \mathrm{~g}$ of KONJAC MANNAN BY ACID HYDROLYSIS (DEGREE OF HYDROLYSIS; 350́)

\begin{tabular}{lccccc}
\hline \multirow{2}{*}{ Sugar } & \multicolumn{2}{c}{ Calculated } & \multicolumn{2}{c}{ Experimental } \\
\cline { 2 - 3 } \cline { 5 - 6 } & yield $(\mathrm{g})$ & ratio & & yield $(\mathrm{mg})$ & ratio \\
\hline $\mathrm{G} \rightarrow \mathrm{M}$ & 9.04 & 1.00 & 2400 & 1.00 \\
$\mathrm{G} \rightarrow \mathrm{G}$ & 1.44 & 0.16 & 300 & 0.13 \\
$\mathrm{M} \rightarrow \mathrm{M}$ & 8.08 & 0.88 & 1400 & 0.58 \\
$\mathrm{M} \rightarrow \mathrm{G}$ & 2.96 & 0.33 & 400 & 0.17 \\
\end{tabular}

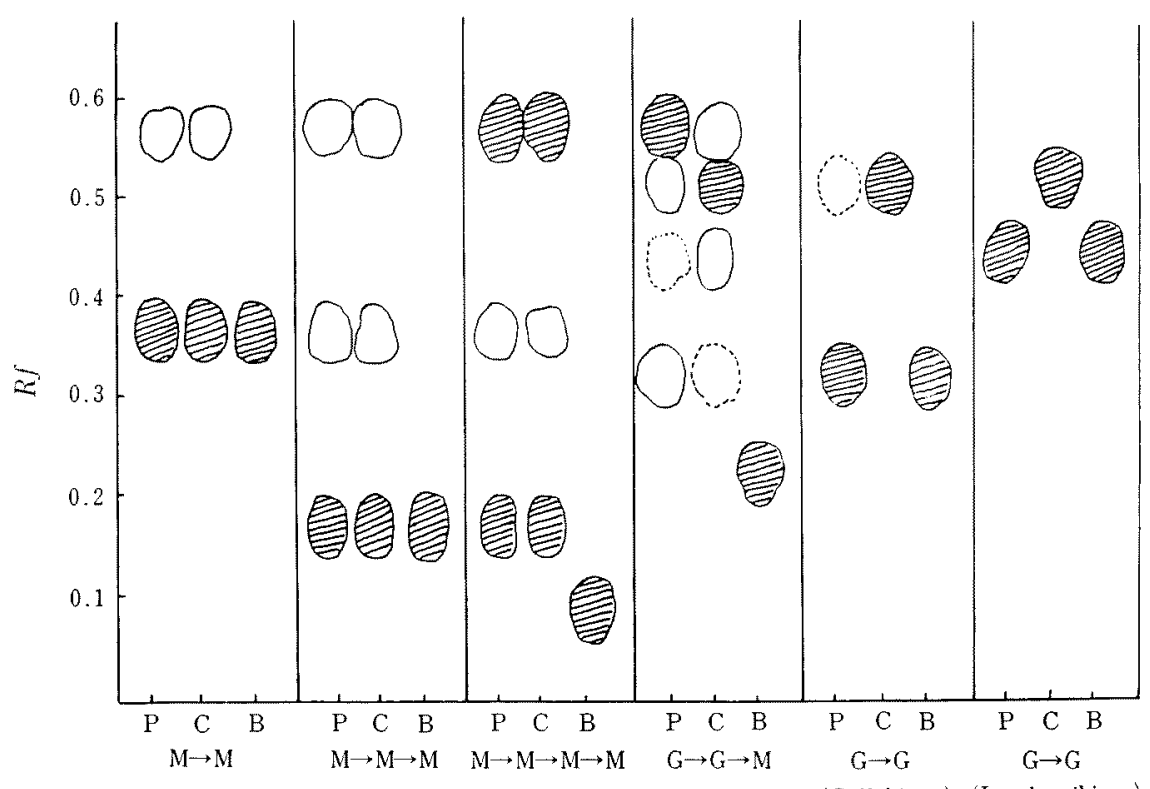

FIG. 3. Action of the Cellulase on the Some Oligosaccharides.

P: Cellulase [Meiji]; C: Meicelase-P; B: Control. 
Table II. Yields of Oligosaccharides Obtained From $50 \mathrm{~g}$ of KonJaC MANNAN BY MeICELASE-P (DEGREE OF HYDROLYSIS; $63 \%$ )

Mole ratio:

\begin{tabular}{|c|c|c|}
\hline \multicolumn{3}{|c|}{$\frac{[\mathrm{B}]+[\mathrm{C}]+[\mathrm{D}]}{[\mathrm{A}]}=\frac{1.03}{1.00}$} \\
\hline Sugar & Yield $(g)$ & mmole \\
\hline $\mathrm{M} \rightarrow \mathrm{M}$ & 3.20 & 9.3 \\
\hline$M \rightarrow M \rightarrow M$ & 2.72 & 5.4 \\
\hline$M \rightarrow M \rightarrow M \rightarrow M$ & 0.76 & 1.1 \\
\hline $\mathrm{M} \rightarrow \mathrm{G}(\mathrm{A})$ & 5.58 & 16.3 \\
\hline$M \rightarrow M \rightarrow G(B)$ & 7.00 & 14.0 \\
\hline $\mathrm{M} \rightarrow \mathrm{M} \rightarrow \mathrm{M} \rightarrow \mathrm{G} \quad(\mathrm{C})$ & 1.33 & 2.0 \\
\hline $\mathrm{M} \rightarrow \mathrm{M} \rightarrow \mathrm{M} \rightarrow \mathrm{M} \rightarrow \mathrm{G}(\mathrm{D})$ & 0.65 & 0.8 \\
\hline
\end{tabular}

with the enzyme. $\mathrm{M} \rightarrow \mathrm{M}$ and $\mathrm{M} \rightarrow \mathrm{M} \rightarrow \mathrm{M}$ were scarcely hydrolyzed by the enzyme, while $\mathrm{M} \rightarrow \mathrm{M} \rightarrow \mathrm{M} \rightarrow \mathrm{M}$ was completely hydrolyzed to give $\mathrm{M} \rightarrow \mathrm{M} \rightarrow \mathrm{M}$ and $\mathrm{M}$ (D-mannose) as main products. From this result, it is shown that the enzyme have abilities of splitting $\beta-1,4$ glucosidic linkage in cellulose as well as $\hat{\beta}-\mathrm{D}$-mannosidic linkage in the manno-oligosaccharides having over four of DP from the non-reducing end. Therefore, the following presumption is possible;

If the repeating unit of the glucomannan is formulated as mentioned before, then at first ß-D-glucosidic linkage will be hydrolyzed by the enzyme, and consequently, $\mathrm{M} \rightarrow \mathrm{M} \rightarrow \mathrm{M} \rightarrow$ $\mathrm{M} \rightarrow \mathrm{G}(\mathrm{A}), \mathrm{M} \rightarrow \mathrm{G}(\mathrm{B})$ and $\mathrm{G}$ (D-glucose) will be obtained. (B) will be scarcely hydrolyzed as like as $\mathbf{M} \rightarrow \mathbf{M}$. Nevertheless, (A) will be affected by the enzyme until the degree of polymerization reaches three. This presumption will result in the isolation of $M \rightarrow M \rightarrow G$ and $\mathrm{M} \rightarrow \mathrm{G}$ in higher yields than the other oligosaccharides, and in expectations that the mole ratio of these oligosaccharides is almost unity. This idea is supported by experimental yields of their sugars (see Table II). ${ }^{2 !}$ Moreover, the above assumption shows that five glycosidic linkages out of eight linkages in the repeating unit of the glucomannan are quickly hydrolyzed by the enzyme. This would be suggested by the observation that the curve of degree of hydrolysis by cellulase declined at about $60.6(\equiv 5 / 8) .{ }^{21}$

Above presumption cannot explain the isolation of the oligosaccharides containing Dmannose residue only. Liberation of these saccharides may signify that the enzyme has also endo-mannanase activity.

\section{EXPERIMENTAL}

Oligosaccharides used were the controlled degradation products of konjac mannan. ${ }^{1,2}$ Paper chromatographic analysis of the sugar was carried out by double ascending method on Toyo No. 2 filter paper using the solvent $n$-butanol : pyridine: water $(6: 3: 4) .{ }^{41}$ The position of the compounds on the paper chromatogram was located by spraying with aniline hydrogen phthalate in $n$-butanol.51

Rate-constants of $\beta$-p-mannosidic and $\beta$ - - -glucosidic linkages. The sugars $(G \rightarrow G, G \rightarrow M, M \rightarrow M$ and $M \rightarrow G$, Ca. $2 \mathrm{mg}$ ) were dissolved in $0.1 \mathrm{~N}$ sulfuric acid $(10 \mathrm{ml})$ and refluxed in a boiling water bath, respectively. Aliquots $(1 \mathrm{ml})$ of reaction mixture were withdrawn at suitable intervals of time and neutralized with $0.1 \mathrm{~N}$ sodium hydroxide $(1.1 \mathrm{ml})$. Thereafter, the solution was diluted with water to $3 \mathrm{ml}$, and $2.5 \%$ sodium borohydride in $0.01 \mathrm{~N}$ sodium hydroxide $(0.2 \mathrm{ml})$ was added and kept at room temperature overnight. The solution was acidified with $5 \mathrm{~N}$ sulfuric acid $(0.8 \mathrm{ml})$ and examined by Anthrone method.6i Since optical density of the sample corresponds to the amount of unhydrolyzed sugar, the ratio of disaccharide survived hydrolysis at each reaction time to that at reaction time 0 is shown as the ratio of each optical density. Therefore, the degree of hydrolysis is shown as follows;

Degree of hydrolysis $=\left(1-\frac{\text { O.D. at time } t}{\text { O.D. at time } 0}\right) \times 100(0)$

Since the acid hydrolysis of glycosidic linkage is a first-order reaction, rate-constants of each disaccharide are calculated from the following equation;

$$
\begin{aligned}
\text { Rate-constants } & =\frac{1}{t}\left\{\ln (\text { sugar })_{0}-\ln (\text { sugar }) t\right. \\
& =\frac{2.303}{t}\left\{2-\log \left(\frac{\text { O.D. at time } t}{\text { O.D. at time } 0}\right)\right\}
\end{aligned}
$$


The results were $0.230 \mathrm{hr}^{-1}$ for $\mathrm{M} \rightarrow \mathrm{M}$ and $\mathrm{M} \rightarrow \mathrm{G}$, and $0.092 \mathrm{hr}^{-1}$ for $\mathrm{G} \rightarrow \mathrm{G}$ and $\mathrm{G} \rightarrow \mathrm{M}$ (Fig. 4). Namely, $\beta$-D-mannosidic linkage is hydrolyzed 2.5 time as fast as $\beta$-D-glucosidic linkage.

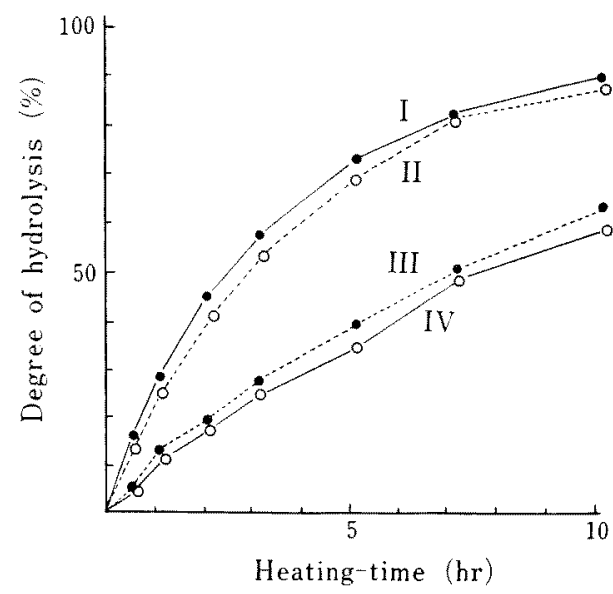

FIG. 4. Acid Hydrolysis of the Disaccharides.

I. $\mathrm{M} \rightarrow \mathrm{G}$ (Mannosylglucose)

II. $\mathrm{M} \rightarrow \mathrm{M}$ (Mannobiose)

III. $\mathrm{G} \rightarrow \mathrm{G}$ (Cellobiose)

IV. $\mathrm{G} \rightarrow \mathrm{M}$ (Epicellobiose)
Action of the cellulase on the manno-oligosaccharides. The sugars $(\mathrm{M} \rightarrow \mathrm{M}, \quad \mathrm{M} \rightarrow \mathrm{M} \rightarrow \mathrm{M}, \quad \mathrm{M} \rightarrow \mathrm{M} \rightarrow \mathrm{M} \rightarrow \mathrm{M}$, $\mathrm{G} \rightarrow \mathrm{G} \rightarrow \mathrm{M}$ and $\mathrm{G} \rightarrow \mathrm{G}$, Ca. $5 \mathrm{mg}$ ) were dissolved in $0.1 \mathrm{M}$ acetate buffer $(\mathrm{pH} 4.5,0.2 \mathrm{ml})$, respectively. To this was added $15 \mu 1$ of the enzyme solution ( $1 \mathrm{mg}$ of cellulase [Meiji] and Meicelase-P in $0.1 \mathrm{ml}$ of the buffer, respectively) and the mixture was kept at $50^{\circ} \mathrm{C}$ for $24 \mathrm{hr}$ after adding a small quantity of toluenc. The reaction products were examined on paper chromatogram after the mixture being heated at $80^{\circ} \mathrm{C}$ for $30 \mathrm{~min}$. The results are shown in Fig. 3 .

\section{REFERENCES}

1) K. Kato and K. Matsuda, Agr. Biol. Chem., 33, 1446 (1969).

2) K. Kato, T. Watanabe and K. Matsuda, ibid., 34, 532 (1970).

3) K. Freudenberg and G. Blomqvist, Ber., 68, 2070 (1935).

4) D. French, D. W. Knapp and J. H. Pazur, J. Am. Chem. Soc., 72, 5150 (1950).

5) S. M. Partridge, Nature, 164, 443 (1949).

6) R. J. Dimler, W. C. Shafer, C. S. Wise and C. E. Rist, Anal. Chem., 24, 1411 (1952). 